\title{
Islet transplants in diabetic Lewis rats prevent and reverse diabetes-induced increases in vascular permeability and prevent but do not reverse collagen solubility changes
}

\author{
J.R. Williamson ${ }^{1}$, K. Chang ${ }^{1}$, E. Rowold ${ }^{1}$, C. Kilo ${ }^{2}$ and P. E. Lacy ${ }^{1}$ \\ Departments of ${ }^{1}$ Pathology and ${ }^{2}$ Internal Medicine, Washington University School of Medicine, St. Louis, Missouri, USA
}

\begin{abstract}
Summary. The effects of islet transplantation on diabetes-induced increases in vascular permeability and collagen solubility were examined in new granulation tissue vessels and collagen formed after induction of streptozotocin diabetes in male Lewis rats. Albumin permeation was increased by $50 \%$ $(p<0.001)$ and collagen solubility was decreased by $50 \%$ $(p<0.001)$ in granulation tissue from untreated diabetic animals as compared with controls. The islet transplants reversed diabetes-induced vascular permeability increases in tissues formed prior to islet transplantation (tissue to blood isotope ratio $=2.1 \pm 0.1$ - SD for controls, $3.2 \pm 0.2$ for diabetic rats and $2.0 \pm 0.2$ for diabetic rats given islets) and prevented permeability increases in new tissues formed following transplantation (tissue to blood isotope ratio $=2.1 \pm 0.1$ for controls,
\end{abstract}

$3.3 \pm 0.8$ for diabetic rats and $1.9 \pm 0.2$ for diabetic rats given islets). In contrast, while islet transplants prevented diabetesinduced decreased collagen solubility in tissues formed after transplantation (controls $=24 \%$, diabetic rats $=12 \%$, and diabetic rats given islets $=24 \%$ ), collagen solubility in tissues formed prior to islet transplantation was virtually unaffected. These findings indicate that collagen changes induced by the diabetic milieu are not nearly as readily reversed by normalization of the diabetic milieu as (diabetes-induced) alterations in vascular functional integrity.

Key words: Islet transplants, diabetes, vascular permeability, collagen crosslinks, nonenzymatic glycosylation
We have recently developed an animal model for investigating the pathogenesis of diabetic vascular disease in which a $50 \%$ increase in permeation of new "granulation tissue" vessels by ${ }^{125}$ I-albumin is demonstrable 3 weeks after the onset of diabetes [1]. Solubility of type I collagen in the new granulation tissue also is markedly decreased [2], implying increased crosslinking. The present experiments were undertaken to investigate the preventability and the reversibility of these changes in diabetic Lewis rats given homologous islet transplants.

\section{Materials and methods}

Three groups of male Lewis rats weighing $275-300 \mathrm{~g}$ were prepared as follows. Group I served as controls and received injections of the diluent utilized for the streptozotocin administered to Groups II and III. Rats in Groups II and III were made diabetic by injection of streptozotocin $(65 \mathrm{mg} / \mathrm{kg})$. At the same time, sterile pieces of polyester fabric were implanted under the skin on the dorsolateral aspect of the thigh of all animals (Groups I-III) as described by Chang et al. [2]. Three weeks later Group III animals received islet transplants [3-6], and animals in all groups received a second fabric implant on the contralateral thigh. Islets were isolated by the collagenase technique [3, 4], sepa- rated on a Ficoll gradient [5] and hand-picked with the aid of a dissecting microscope. Approximately 1500 islets were transplanted into the liver of each diabetic animal via the portal vein [6]. Six weeks after the onset of diabetes, vascular permeation by ${ }^{125} \mathrm{I}$-albumin was assessed in tissues in the first (6-week-old) and in the second (3-weekold) fabric implants, as well as in several additional tissues.

In Group III rats, the 6 week fabric implant tissue was subjected to the full impact of the diabetic milieu for the first 3 weeks, and to a rapidly normalizing metabolic milieu during the second 3 weeks following islet transplantation. In contrast, the 6 week implants in Groups I and II were subjected to normal and diabetic milieus respectively for the entire 6 weeks. The tissue formed in the second fabric implant (inserted at the time of islet transplantation) was subjected to a normal milieu for 3 weeks in Group I, to a diabetic milieu for 3 weeks in Group II, and to a rapidly normalizing diabetic milieu in Group III.

The techniques for assessment of albumin permeation of vessels and solubility of collagen have been published and will be described only briefly $[1,2,7]$. Assessment of vascular permeation by albumin is based on quantification of ${ }^{125} \mathrm{I}$-albumin, ${ }^{51} \mathrm{Cr}$-RBC, and ${ }^{57} \mathrm{Co}$-EDTA in blood and tissues sampled $6.5-8 \mathrm{~min}$ after intravenous injection of the tracers $[1,7]$. An index of albumin permeation, the tissue to blood isotope ratio (TBIR-I/Cr), is derived from the formula:

TBIR $=\frac{\left({ }^{125} \mathrm{I}-\mathrm{BSA}{ }^{51} \mathrm{Cr}-\mathrm{RBC}\right)-\text { Tissue }}{\left({ }^{125} \mathrm{I}-\mathrm{BSA}{ }^{51} \mathrm{Cr}-\mathrm{RBC}\right)-\mathrm{Blood}}$.

A TBIR-I/Cr greater than 1 indicates that the volume of distribution of albumin relative to that of erythrocytes is greater in the tissue than 
in large vessels and is indicative of permeation of the vasculature by albumin into the extravascular space. Since the capillary hematocrit is somewhat lower than that of large vessels [7], the TBIR-I/Cr is always slightly greater than 1 . While the terms 'increased vascular permeability' and 'increased vascular permeation by albumin' are sometimes used interchangeably, it should be understood that these two terms are not exactly synonymous. Permeability refers to the porosity (i.e. pore size, number, and surface area) of the vessel wall, whereas permeation refers to the movement of a tracer molecule, i. e. ${ }^{125} \mathrm{I}$-albumin, across the vessel wall. While an increase in the number and/or size of vascular pores large enough to accommodate albumin will be manifested by an increase in albumin permeation, an increase in albumin permeation also may occur as a consequence of haemodynamic changes leading to an increase in pressure gradients across vessel walls. Such changes would increase the filtration (permeation) of plasma water and proteins (including albumin) through existing pores without any change in porosity of the vessel wall. At the present time we do not know the mechanism(s) responsible for the diabetes-induced increase in albumin permeation of vessels in this model.

The TBIR.- ${ }^{57} \mathrm{Co}-\mathrm{EDTA}{ }^{51} \mathrm{Cr}-\mathrm{RBC}$ (TBIR-Co/Cr) also is determined. Since ${ }^{57} \mathrm{Co}$-EDTA is a much smaller molecule than ${ }^{125} \mathrm{I}$-BSA, it permeates vessel walls much more readily and accesses virtually the entire extracellular space. If only the TBIR-I/Cr is determined and it is low or close to 1 , it is not possible to know whether the low value is because of the permeability characteristics of the vessel or because the interstitial fluid space is so small that it is limiting, or because blood flow was so low that little albumin was available to access the interstitial fluid space. On the other hand, a low TBIR-I/Cr in the face of a high TBIR- $\mathrm{Co} / \mathrm{Cr}$ indicates that the permeability characteristics of the vessel wall limited permeation of the vessel by ${ }^{125}$ I-BSA. TBIR$\mathrm{Co} / \mathrm{Cr}$ values in granulation tissue were $\sim 40 \mathrm{x}$ TBIR-I/Cr values.

Table 1. Vascular permeation by ${ }^{125}$ I-BSA in 3-week old tissue formed during the 3-week period following islet transplantation in Group III diabetic rats versus 6 week old tissue formed during the 3 weeks prior to and the 3 weeks after islet transplantation

\begin{tabular}{clll}
\hline & $(n=)$ & 3-week tissue & 6-week tissue \\
\hline I Control rats & $(7)$ & $2.09 \pm 0.11^{\mathrm{c}}$ & $2.08 \pm 0.14$ \\
II Diabetic rats & $(6)$ & $3.26 \pm 0.75^{\mathrm{d}}$ & $3.22 \pm 0.15^{\mathrm{d}}$ \\
III Diabetic rats +islets & $(6)$ & $1.92 \pm 0.23$ & $2.00 \pm 0.23$ \\
\hline
\end{tabular}

a In all 3 groups of animals albumin permeation was assessed in fabric implanted at the same time that islets were transplanted into Group III. ${ }^{b}$ Albumin permeation was assessed in tissues formed in fabric implanted 6 weeks earlier in all 3 groups. In Group III the tissue was subjected to a diabetic milieu during the first 3 weeks and to a rapidly normalizing metabolic milieu during the last 3 weeks following islet transplantation. ${ }^{c}$ Mean \pm SD of the tissue to blood isotope ratio, an index of albumin permeation of the vasculature (see text for details of calculation). ${ }^{d}$ Significantly different from controls and from diabetic animals receiving islet transplants $(p<0.001)$
The blood volume of newly formed granulation tissue, expressed as $\mathrm{ml} / \mathrm{g}$ of implanted fabric, is derived from the formula: ${ }^{51} \mathrm{Cr} \mathrm{cpm}$ per $\mathrm{g}$ fabric $/{ }^{51} \mathrm{Cr} \mathrm{cpm}$ per $\mathrm{ml}$ whole blood as described by Kilzer et al. [7]

\section{Assessment of collagen crosslinking and nonenzymatic glycosylation of granulation tissue}

Since solubility of collagen in neutral salt solutions and dilute acids is inversely related to the extent of intermolecular crosslinks [8], we have quantified collagen solubility in $0.5 \mathrm{~mol} / \mathrm{l}$ acetic acid as an index of collagen crosslinking [2]. The implant tissue is first finely minced with scissors and a razor blade, and extracted with $0.15 \mathrm{~mol} / 1 \mathrm{NaCl}$ in $50 \mathrm{mmol} / 1$ Tris (at neutral $\mathrm{pH}$ ) at $4{ }^{\circ} \mathrm{C}$ for $4-6 \mathrm{~h}$ to wash out plasma proteins and other soluble noncollagenous proteins. The minced tissue is then centrifuged and the residue is extracted at $4{ }^{\circ} \mathrm{C}$ in $0.5 \mathrm{~mol} / 1$ acetic acid ( $1 \mathrm{ml}$ per $100 \mathrm{mg}$ wet weight of implant) for $48 \mathrm{~h}$ with constant shaking. After centrifugation at $37,000 \mathrm{~g}$ for $60 \mathrm{~min}$, the supernatant and pellet are lyophylized and then hydrolyzed in $0.3 \mathrm{~N}$ oxalic acid for $2 \mathrm{~h}$ at $100^{\circ} \mathrm{C}$. The samples are then cooled to room temperature and proteins are precipitated by addition of trichloroacetic acid (TCA). Nonenzymatically bound hexoses in the supernatants are measured as 5-hydroxymethylfurural by the thiobarbituric acid method [9]. The TCA precipitates are lyophylized and hydrolyzed in $6 \mathrm{~N}$ $\mathrm{HCl}$ for $24 \mathrm{~h}$ at $100^{\circ} \mathrm{C}$ for determination of hydroxyproline [10] and total protein [11]. Soluble and insoluble collagen are estimated from the hydroxyproline content of the supernatant and pellet respectively using a correction factor of 10 (based on evidence that hydroxyproline accounts for $\sim 10 \%$ of the weight of type I collagen [12]). Total collagen is considered equal to the sum of the soluble and insoluble fractions.

Plasma glucose levels are determined by the glucose oxidase method [13].

\section{Statistical analyses}

The statistical significance of differences between tissues in the same animal was assessed by the paired Student's t-test; differences between animal groups were assessed by the standard Student's t-test.

\section{Results}

Albumin permeation in new granulation tissue vessels in untreated diabetic rats was increased by $\sim 50 \%(p<$ 0.001 ) and was virtually identical in 3-week and 6-week implants (Table 1). In contrast, these increases in albumin permeation were completely prevented and reversed in the 3-week and 6-week implants, respectively, in diabetic rats receiving islet transplants (Table 1 ).

Table 2. Collagen content and solubility in $0.5 \mathrm{~mol} / 1$ acetic acid in 3-week-old tissue formed during the 3-week period following islet transplantation in Group III versus that of 6-week-old tissue formed during the 3 weeks prior to and the 3 weeks after islet transplantation

\begin{tabular}{|c|c|c|c|c|c|}
\hline & \multirow{2}{*}{$(n=)$} & \multicolumn{2}{|c|}{3 -week tissue ${ }^{a}$} & \multicolumn{2}{|c|}{ 6-week tissue ${ }^{b}$} \\
\hline & & Content $^{c}$ & Solubility $^{d}$ & Content & Solubility \\
\hline I Control rats & (7) & $74 \pm 5^{e}$ & $23.7 \pm 2.5^{\mathrm{e}}$ & $126 \pm 8^{\mathrm{f}}$ & $17.1 \pm 1.9^{f}$ \\
\hline II Diabetic rats & (6) & $74 \pm 4$ & $11.5 \pm 1.1^{\mathrm{g}}$ & $128 \pm 4^{f}$ & $10.4 \pm 1.5^{\mathrm{g} j \mathrm{j}}$ \\
\hline III Diabetic rats + islets & (7) & $73 \pm 5$ & $24.5 \pm 1.5^{\mathrm{h}}$ & $126 \pm 11^{f}$ & $13.5 \pm 1.2^{\mathrm{i}, \mathrm{f}}$ \\
\hline
\end{tabular}

${ }^{2}$ In all 3 groups collagen content and solubility were assessed in tissues formed in fabric implanted at the same time that islets were transplanted into Group III. ${ }^{b}$ Collagen content and solubility were assessed in tissues formed in fabric implanted 6 weeks earlier in all 3 groups. In Group III the tissue was subjected to a diabetic milieu in the first 3 weeks and to a rapidly normalizing metabolic milieu during the last 3 weeks following islet transplantation. ${ }^{c}$ Collagen content expressed as $\mu \mathrm{g}$ of collagen $/ \mathrm{mg}$ fabric implant. ${ }^{\mathrm{d}}$ Percent of total collagen in the implant tissue that is soluble in $0.5 \mathrm{~mol} / 1$ acetic acid. ${ }^{*}$ Mean $\pm \mathrm{SD}$. ${ }^{\mathrm{f}}$ Significantly different from 3 week tissue $(p<0.001)$. ${ }^{\mathrm{g}}$ Significantly different from controls $(p<$ $0.001) .{ }^{\mathrm{h}}$ Significantly different from untreated diabetic rats $(p<0.001) .{ }^{\mathrm{i}}$ Significantly different from controls and from untreated diabetic rats $(p<0.005) .{ }^{j}$ Significantly different from 3 week tissue $(p<0.025)$ 
Table 3. Blood volume in 3-week-old tissue formed during the 3-week period following islet transplantation in Group III versus 6-week-old tissue formed during the 3 weeks prior to and during the 3 weeks after islet transplantation

\begin{tabular}{clll}
\hline & $(n=)$ & 3-week tissue $^{\mathrm{a}}$ & 6-week tissue $^{\mathrm{b}}$ \\
\hline I Control rats & $(7)$ & $0.043 \pm 0.003^{\mathrm{c}}$ & $0.051 \pm 0.003^{\mathrm{d}}$ \\
II Diabetic rats & $(6)$ & $0.041 \pm 0.005$ & $0.052 \pm 0.004^{\mathrm{d}}$ \\
III Diabetic rats & $(6)$ & $0.042 \pm 0.003$ & $0.047 \pm 0.004^{\mathrm{d}}$ \\
$\quad+$ islets & & & \\
\hline
\end{tabular}

a Blood volume for all 3 groups was assessed in tissues formed in fabric implanted at the same time that islets were transplanted into Group III. ${ }^{b}$ Blood volume was assessed in tissues formed in fabric implanted 6 weeks earlier in all 3 groups. In Group III the tissue was subjected to a diabetic milieu during the first 3 weeks and to a rapidly normalizing metabolic milieu during the last 3 weeks following islet transplantation. ${ }^{\mathrm{c}}$ Blood volume expressed as $\mathrm{ml} / \mathrm{gm}$ fabric. ${ }^{\mathrm{d}}$ Significantly different from 3-week tissue $(p<0.001)$.

Table 4. Nonenzymatic glycosylation (NEG) of 3-week-old granulation tissue formed during the 3-week period after islet transplantation in Group III versus 6-week-old tissue formed during the 3 weeks prior to and during the 3 weeks after islet transplantation

\begin{tabular}{llll}
\hline & $(n=)$ & 3-week tissue & 6-week tissue \\
\hline I Control rats & $(7)$ & $2.13 \pm 0.19^{\mathrm{c}}$ & $2.38 \pm 0.13$ \\
II Diabetic rats & $(6)$ & $2.99 \pm 0.14^{\mathrm{d}}$ & $2.95 \pm 0.14^{\mathrm{d}}$ \\
III Diabetic & $(7)$ & $2.80 \pm 0.11^{\mathrm{d}, \mathrm{e}}$ & $2.72 \pm 0.10^{\mathrm{d}, \mathrm{f}}$ \\
$\quad$ rats +islets & & &
\end{tabular}

a In all 3 groups NEG of granulation tissue was assessed in fabric implanted at the time of islet transplantation into Group III. ${ }^{b}$ NEG was assessed in tissues formed in fabric implanted 6 weeks earlier in all 3 groups. In Group III the tissue was subjected to a diabetic milieu during the first 3 weeks and to a rapidly normalizing metabolic milieu during the last 3 weeks following islet transplantation. "Mean \pm SD (nmol 5-hydroxymethylfurfural/mg granulation tissue protein). ${ }^{d} \mathrm{Sig}$ nificantly different from controls $(p<0.001)$. ${ }^{\text {e Significantly different }}$ from untreated diabetic rats $(p<0.025)$. ${ }^{\mathrm{f}}$ Significantly different from untreated diabetic rats $(p<0.01)$.

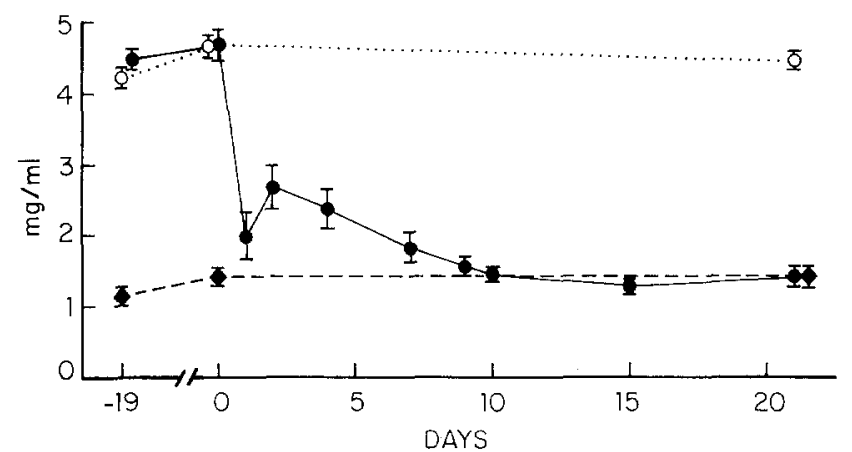

Fig. 1. Plasma glucose values in control $(\diamond)$, untreated diabetic $(O)$, and diabetic rats receiving islet transplants $(-)$ at day 0 . Vertical bars represent standard errors

Collagen solubility in $0.5 \mathrm{~mol} / 1$ acetic acid was markedly decreased (compared to nondiabetic controls, $p<0.001$ ) in both 3-week and 6-week implants of untreated diabetic animals (Table 2). In both controls and untreated diabetic animals, collagen in the 6-week implants was less soluble than that of 3-week implants $(p<0.001)$. Islet transplants completely prevented the decrease in collagen solubility in tissue formed (in the 3-week implants) after islet transplantation; on the other hand, collagen solubility of tissue from the 6-week implants was approximately midway between that of controls and untreated diabetic animals (Table 2).

Total collagen accumulation in 3-week implants was identical in controls, in untreated diabetic animals, and in diabetic animals receiving islet transplants (Table 2); likewise, total collagen accumulation in 6-week fabric implants was identical in all 3 groups. Six week implants, however, contained $\sim 1.7 \times$ as much collagen $(p<0.001)$ as the 3-week implants.

Blood volume also was the same in 3-week implants for all 3 groups (Table 3 ) and was significantly increased by $\sim 23 \%$ in the 6-week implants in the controls and untreated diabetic animals and by $\sim 10 \%$ in the islet transplanted diabetic animals $(p<0.001$ for paired Student's t-tests in all 3 groups).

Nonenzymatic glycosylation of granulation tissue proteins was significantly greater in 3-week tissue of diabetic and islet-transplanted diabetic animals relative to controls $(p<0.001)$ and the values for the islet-transplanted diabetic animals were slightly less than those for the untreated diabetic animals (Table 4). Nonenzymatic glycosylation values for 6 week tissues from nondiabetic controls were $\sim 10 \%$ higher than those for 3 -week tissues, whereas corresponding values for untreated diabetic and islet-transplanted diabetic animals did not differ significantly from those of 3-week implants.

In view of the report of Schleicher and Wieland [14] the thiobarbituric assay may detect (nonreducible) nonketoamine carbohydrate linkages in glycoproteins in addition to reducible ketoamine linkages. It is noteworthy, therefore, that following tritiated borohydride reduction, over twice as much ${ }^{3} \mathrm{H}$-hexosyl-lysine and ${ }^{3} \mathrm{H}$ hexosyl-hydroxylysine are recovered in granulation tissue extracts from diabetic animals as from controls (unpublished results).

Plasma glucose values for all three groups of animals are shown in Figure 1. It can be seen that the plasma glucose values were identical in both diabetic groups at the time of islet transplantation, and that they were virtually normal in the islet-transplanted group $\sim 9$ days after transplantation.

\section{Discussion}

The magnitude of the diabetes-induced increases in albumin permeation in new granulation tissue vessels in this experiment is virtually identical to that observed previously $[1,15,16,17]$. The observation that albumin permeation was normal in both the 3-week and the 6-week implants in islet-transplanted diabetic rats attests to the efficacy of islet transplantation in the prevention and treatment of diabetes-induced vascular 
dysfunction and indicates that: (1) the pathophysiological perturbations and vascular changes responsible for increased albumin permeation in this model are readily reversible (after diabetes of 3 weeks duration) during the 3 weeks following islet transplantation, and (2) normalization of the diabetic milieu by transplanted islets prevents the increase in albumin permeation associated with untreated diabetes. This observation is of particular importance in view of recent findings which suggest that diabetes-induced increases in albumin permeation in this model are due to increased porosity of vascular endothelium rather than to haemodynamic or haemorheological alterations (unpublished observations).

The reversibility of diabetes-induced increases in vascular permeability in this model by islet transplants is consistent with the observations of Krupin et al [18], who reported that islet transplants reversed increased fluorescein accumulation in the anterior chamber and vitreous of diabetic rats. While considerable evidence suggests that increased fluorescein accumulation in the eyes of diabetic rats may reflect retinal pigment epithelial degeneration rather than retinal capillary changes [1], the increased albumin permeation in new granulation tissue vessels would appear to be virtually unattributable to any cause other than compromised functional integrity of vascular endothelial cells.

Recent evidence indicates that vascular permeability increases in this model are linked to increased metabolism of glucose by the enzyme aldose reductase [15]. Since sorbitol production and accumulation is influenced by many factors $[15,17,19]$ including tissue glucose levels, normalization of glucose levels following islet transplantation would be expected to correct imbalances in sorbitol myo-inositol metabolism and associated alterations in vascular permeation by albumin. Unfortunately, no data are available on the effects of islet transplants on tissue levels of sorbitol and myo-inositol in the present experiments.

The observation that diabetes-induced increases in vascular permeability and decreases in collagen solubility are completely prevented in tissues formed after islet transplantation attests to the efficacy of the transplanted islets in normalizing the diabetic milieu. Data from other experiments suggest that the beneficial effect of islet transplantation on collagen solubility is more likely due to correction of hormonal imbalances associated with diabetes than to normalization of plasma glucose levels or to correction of imbalances in sorbitol/ myo-inositol metabolism [15-17]. Thus, inhibitors of aldose reductase which prevent diabetes-induced increases in vascular permeability in this model have no effect on collagen solubility, plasma glucose levels, or nonenzymatic glycosylation $[15,16]$. Castration of male diabetic rats, on the other hand, prevents diabetes-induced decreases in collagen solubility (as well as increased albumin permeation of vessels) but has no effect on plasma glucose levels or nonenzymatic glycosylation of plasma proteins or granulation tissues [17]. These data do not support a role for nonenzymatic glycosylation of vascular constituents as an explanation for diabetes-induced increased albumin permeation in this animal model.

The finding that collagen solubility in tissues from the 6-week implants of islet-transplanted diabetic rats was essentially midway between the values for controls and untreated diabetic rats suggests that the solubility characteristics of collagen formed in the diabetic milieu are not altered by subsequent normalization of glucose levels. Indeed, if it is assumed that: (1) the solubility of all collagen formed in the fabric prior to normalization of plasma glucose levels was $\sim 11.5 \%,(2)$ the solubility of collagen formed after normalization of the diabetic milieu was $\sim 24 \%$, and (3) the rate of collagen formation was relatively constant during the 3 weeks following islet transplantation, then it can be estimated that $\sim 11-12$ days elapsed following islet transplantation before solubility (i.e. crosslinking) of newly synthesized collagen was normal. This time point agrees fairly well with the finding that plasma glucose values were virtually normalized $\sim 10$ days following islet transplantation.

The finding that collagen solubility in the 3-week implants was normal even though plasma glucose levels had not returned to normal until 11-12 days following islet transplantation is consistent with the likelihood that the vast majority of the collagen contained in these implants was formed in the 10-day period after plasma glucose values were normalized. The observation in the nondiabetic group that only $75 \%$ as much collagen was soluble in 6-week implants as in 3-week implants is consistent with the well known age related (normal maturation) increases in nonreducible collagen crosslinks in normal animals [20].

The finding that vascular permeability in 6-week tissue of controls and of islet-transplanted diabetic rats was identical to that in 3-week tissues from the same animals, even though collagen solubility was significantly lower in the 6-week than in the 3-week tissues, suggests that there is no causal relationship between type I collagen solubility (i.e. crosslinking) and vascular permeability increases in this model. This observation is consistent with the effects of sorbinil, discussed above, as well as with the observation that castration of nondiabetic rats has no effect on vascular permeability but causes a marked increase in collagen solubility.

An important clinical implication of these findings is that collagen changes (i.e. increased crosslinking) induced by the diabetic milieu are not nearly as readily reversed as the diabetic milieu itself, or as diabetes-induced increases in vascular permeability. These findings are consistent with the well known relatively long half-life of collagen.

Acknowledgements. This research was supported by National Institutes of Health Grants HL13694 and AM01226 and by the Kilo Diabetes and Vascular Research Foundation. 


\section{References}

1. Kilzer $P$, Chang $K$, Marvel J, Rowold E, Jaudes $P$, Ullensvang $S$, Kilo C, Williamson JR (1985) Albumin permeation of new vessels is increased in diabetic rats. Diabetes $34: 333-336$

2. Chang K, Uitto J, Rowold EA, Grant GA, Kilo C, Williamson JR (1980) Increased collagen crosslinkages in experimental diabetes: Reversal by B-aminopropionitrile (BAPN) and D-penicillamine. Diabetes 29: 778-781

3. Lacy PE, Kostianovsky M (1967) A method for the isolation of intact islets of Langerhans from the rat pancreas. Diabetes 16:35-39

4. Lacy PE, Walker MM, Fink CJ (1972) Perifusion of isolated rat islets in vitro. The participation of the microtubular system in the biphasic release of insulin. Diabetes 21:987-998

5. Lindall A, Steffes M, Sorenson R (1969) Immunoassayable insulin content of subcellular fractions of rat islets. Endocrinology 85 : $218-223$

6. Kemp CB, Knight MJ, Scharp DW, Ballinger WF, Lacy PE (1973) Effect of transplantation site on the results of pancreatic islet isografts in diabetic rats. Diabetologia 9:486-491

7. Kilzer P, Chang K, Marvel J, Kilo C, Williamson JR (1985) Tissue differences in vascular permeability changes induced by histamine. Microvasc Res 30:270-285

8. Miller EJ, Rhodes RK (1982) Preparation and characterization of the different types of collagen. In: Cunningham LW, Frederiksen DW (eds) Methods in enzymology, Vol 82: Structural and contractile proteins. Part A. Extracellular matrix. Academic Press, New York, pp 33-64

9. Fluckiger R, Winterhalter KH (1976) In vitro synthesis of hemoglobin $A_{1 c}$. FEBS Lett 71:356

10. Kivirikko KI, Laitinen O, Prockop DJ (1967) Modifications for a specific assay of hydroxyproline in urine. Anal Biochem 19: 249-255

11. Moore S, Stein WH (1954) A modified ninhydrin reagent for the photometric determination of amino acids and related compounds. J Biol Chem 211: 907-913

12. Berg RA (1982) Determination of 3- and 4-hydroxyproline. In: Cunningham LW, Frederiksen DW (eds) Methods in enzymology, Vol 82. Structural and contractile proteins. Part A. Extracellular matrix. Academic Press, New York, pp 372-398
13. Huggett A, Mixon DA (1957) Use of glucose oxidase, peroxidase and 0 -dianisidine in determination of blood and urinary glucose. Lancet 2: 368-379

14. Schleicher E, Wieland OH (1981) Specific quantification by HPLC of protein (lysine) bound glucose in human serum albumin and other glycosylated proteins. J Clin Chem Clin Biochem 19: $81-87$

15. Williamson JR, Chang $\mathrm{K}$, Rowold E, Marvel J, Tomlinson M, Sherman WR, Ackermann KE, Kilo C (1985) Sorbinil prevents diabetes-induced increases in vascular permeability but does not alter collagen crosslinking. Diabetes 34: 703-705

16. Williamson JR, Chang K, Rowold E, Marvel J, Tomlinson M, Sherman WR, Ackermann KE, Kilo C (1986) Diabetes-induced increases in vascular permeability and changes in granulation tissue levels of sorbitol, myo-inositol, chiro-inositol, and scyllo-inositol are prevented by sorbinil. Metabolism (in press)

17. Williamson JR, Rowold E, Chang K, Marvel J, Tomlinson M, Sherman WR, Ackermann KE, Berger RA, Kilo C (1986) Sex steroid-dependency of diabetes-induced changes in polyol metabolism, vascular permeability and collagen crosslinking. Diabetes 35: $20-27$

18. Krupin T, Waltman SR, Scharp DW, Oestrich C, Feldman SD, Becker B, Ballinger WF (1979) Ocular fluorophotometry in streptozotocin diabetes mellitus in the rat: effect of pancreatic islet isografts. Invest Ophthalmol Vis Sci 18: 1185-1190

19. Varma SD, Kinoshita JH (1974) Sorbitol pathway in diabetic and galactosemic rat lens. Biochim Biophys Acta 338: 632-640

20. Bailey AJ, Robins SP, Balian G (1974) Biological significance of the intermolecular crosslinks of collagen. Nature 251: 105-109

Received: 18 November 1985

and in revised form: 18 March 1986

Dr. Joseph R. Williamson

Washington University School of Medicine

Department of Pathology (Box 8118)

660 S. Euclid Avenue

St. Louis, MO 63110

USA 